\section{Reliability of vitreous histological detection of pathogenic fungi in the diagnosis of fungal endophthalmitis}

Introduction

Fungal endophthalmitis is a potentially devastating intraocular infection that is commonly diagnosed in apparently healthy and immunocompromised individuals worldwide. ${ }^{1}$ Accurate identification and prompt therapy is essential to ensure a positive outcome to this often devastating disease. In most cases of fungal endophthalmitis, diagnosis can be made based on patient medical history, clinical appearance, and basic investigation. . $^{2,3}$ However, conventional diagnostic techniques and investigative procedures, such as smear or culture, from intraocular fluids (aqueous humor or vitreous) can suffer from poor sensitivity (25-60\%), which may make diagnoses both difficult and time consuming. ${ }^{4,5}$

Advances in surgical vitrectomy and pathological techniques of vitreous specimens have expanded the options for diagnoses of intraocular malignancies and endophthalmitis. ${ }^{6}$ In this study, we report the histopathological detection results in vitreous biopsy fluid from patients who underwent diagnostic vitrectomy for clinically suspected fungal endophthalmitis. To our knowledge, this is the first study to investigate histopathological testing of fungal from vitreous fluid in a series of patients.

Materials and methods

Patients and surgical procedures

From March 2010 to December 2010, we consecutively enrolled 10 clinically suspected fungal endophthalmitis cases among 10 patients in this prospective study, which was conducted
Acceived. 7 May 2014

9 October 2014

Published online:

14 November 2014
Eye (2015) 29, 424-427; doi:10.1038/eye.2014.278;

published online 14 November 2014 
at our hospital and included seven males and three females between the ages of 23-60 years (Table 1). After informed consent was obtained from all patients, nondiluted vitreous fluid samples were collected $(0.5-1.0 \mathrm{ml})$ during vitrectomy procedures by one surgeon for diagnostic and therapeutic purposes. At the time of sampling, all patients displayed active intraocular infections.

A standard three-port pars plana vitrectomy approach was performed. Manual aspiration and specimen collection were carried out for retrieval of pure vitreous into a $5-\mathrm{ml}$ syringe connected to a hand piece until the eye was noted to visibly soften, and then infusion began. The vitreous was aspirated into a sterilized syringe. ${ }^{7}$ The remaining infused vitreous was kept for histological evaluation.

\section{Sample preparation}

Intraocular samples were immediately transported to the pathology laboratory at room temperature. The biopsied materials are divided into three portions: for direct smear examination, for cultures of fungi, and for histological detection.

For vitreous sample smears, specimens were placed directly on the slides from the tapping syringes and needles, and then detected under a microscope for the appearance of fungi. For fungal and bacterial cultures, vitreous specimens were processed within $1 \mathrm{~h}$ of sample collection according to standard techniques and retained for at least 2 weeks for fungi identification.

\section{Histological testing of fungi}

For histological evaluation, the vitreous samples were concentrated by centrifugation at 3000-4000 $g$ at room temperature for $6-8 \mathrm{~min}$. The precipitates at the bottom of the tube following centrifugation were mixed with human or animal serum to form clumps. The specimens were then fixed in $4 \%$ formalin overnight, dehydrated, and embedded in paraffin before sectioning. Routine hematoxylin and eosin (H\&E) and periodic-acid Schiff (PAS) staining were performed according to standard techniques. In addition, for direct examination of the samples, Gram, Gomori methenamine-silver (GMS), and Giemsa staining were applied. All slides were reviewed by an ophthalmic pathologist.

\section{Results}

Among the 10 cases with a suspected diagnosis of fungal endopthalmitis, seven $(7 / 10)$ were confirmed by specific histopathological detection of pathogenic fungi and four $(4 / 10)$ were confirmed through culture with a sensitivity of $40 \%$. The other five cases (5/10) were confirmed by smear $(50 \%)$ of the vitreous biopsy samples (Table 1 ).

Among the seven positive results by histological detection, the fungi was found in six cases by H\&E and PAS staining (Figure 1), whereas only one case required GMS staining to confirm fungal infection.

\section{Discussion}

Fungal endophthalmitis is an infrequent but sightthreatening disease, which can be classified as endogenous fungal endophthalmitis (EFE) or exogenous fungal endophthalmitis (EXFE) depending upon the mode of infection. EFE is usually caused by endogenously acquired fungi, whereas EXFE is caused by the traumatic implantation of fungal pathogens. Among the 10 fungal endophthalmitis patients in our study, four had a history of intravenous drug abuse and five had trauma history.

Table 1 Fungal endophthalmitis: smear, culture, histological results, and etiology

\begin{tabular}{lcclccc}
\hline $\begin{array}{l}\text { Patient } \\
\text { no. }\end{array}$ & $\begin{array}{c}\text { Age } \\
\text { (years) }\end{array}$ & Sex & Etiology & Smear & Culture & $\begin{array}{c}\text { Histological } \\
\text { detection }\end{array}$ \\
\hline 1 & 23 & M & Trauma & - & + & - \\
2 & 45 & F & IVDU & + & - & + \\
3 & 52 & M & IVDU & - & - & - \\
4 & 48 & M & IVDU & - & + & + \\
5 & 60 & M & After removal & + & - & + \\
& & & of the bladder & & & + \\
& & & tumor & & & + \\
6 & 42 & M & Trauma & + & - & + \\
7 & 25 & F & Trauma & - & - & + \\
9 & 32 & F & Trauma & + & + & + \\
10 & 39 & M & IVDU & + & - & + \\
\hline & 44 & F & Trauma & - & + & + \\
\hline
\end{tabular}

Abbreviation: IVDU, intravenous drug abuse.

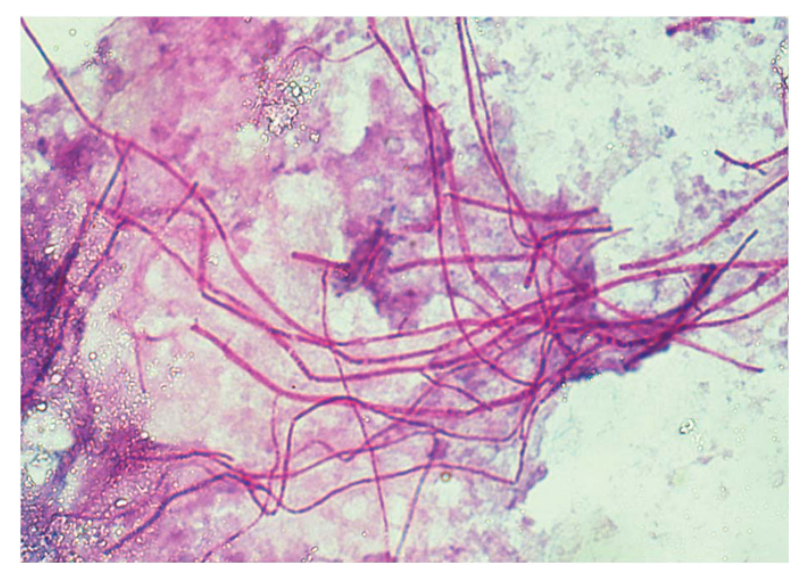

Figure 1 Histological image of PAS staining from a vitreous specimen. A number of PAS-positive filamentous fungi were observed, indicating fungal infection $(\times 400)$. 
Prognosis depends on the virulence of the organism and intervention timing. Early, adequate treatment can prevent these infectious agents from causing irreversible ocular damage. Unfortunately, most outcomes of fungal endophthalmitis are poor, likely due to the prolonged time to diagnosis. Diagnostic delay might be explained by the rarity of fungal endophthalmitis and the subacute nature of early infection, or the difficulty of distinguishing true infection from the occasionally observed postprocedural sterile inflammation. ${ }^{8}$

The microbiological diagnosis of fungal endophthalmitis is usually based on microscopy and culture of the microorganisms in vitreous fluid. Smears made from these samples may be examined immediately after detecting the fungi by direct microscopy. Although smear examination is a rapid method, it suffers from low sensitivity; the positive ratio for fungal smear in our study was $50 \%$. Culture provides a gold standard diagnostic test for fungi, but suffers from poor sensitivity, which may be related to its slow growth rate or requiring fastidious growth conditions; culturing vitreous samples for the fungi had a sensitivity of only $40 \%$ in our study. It has been previously reported that fungal cultures can be positive in $44-70 \%$ of patients diagnosed clinically. ${ }^{9}$ In addition, the culture must be kept at the laboratory for at least 4-6 weeks to ensure that slow-growing or fastidious fungal organisms are not missed. Despite the application of microbiological techniques and immediate processing of samples, the sensitivity of conventional methods to detect organisms in vitreous fluid range from 25 to $60 \%{ }^{10}$ Testing intraocular fluid by smear or culture to identify fungal pathogens can be extremely useful, but suffers from poor sensitivity. An accurate clinical diagnosis of this infectious eye disease, confirmed by laboratory techniques, is crucial for appropriate treatment to reduce significant visual loss caused by all forms of fungal endophthalmitis.

As the sensitivity of conventional fungal cultures is not high, and the culture growth rates are slow, histopathologic investigation on vitreous samples is a method to improve this sensitivity and to identify fungal endophthalmitis.

Vitrectomy samples are more sensitive for fungal detection than vitreous needle biopsies, as vitreous humor provides the best samples for histopathology of fungal pathogens. Furthermore, it has been reported that undiluted vitreous has a higher yield of confirmed positive cultures than aqueous humor. ${ }^{11}$ During histological procedures, most vitreous could be permanently fixed and examined under a microscope, which yields higher concentration of pathogens and sensitivity than vitreous smear and culture.
Detection of granulomatous inflammation in vitreous samples by routine H\&E staining may be associated with fungal infection. When fungal pathogens are observed directly or by PAS staining, fungal endophthalmitis is diagnosed. GMS and Giemsa staining can also be used for fungal detection. Furthermore, histopathologic investigation only requires a few steps and is not time intensive, which makes it an appropriate technique for primary hospitals.

Despite a high sensitive ratio for fungal detection, histopathologic investigations have limited roles in the identification of the exact fungal species. However, fungal morphological features and special staining or immunohistochemistry can be helpful to generate preliminary judgments.

Recently, polymerase chain reaction (PCR) was introduced as a diagnostic tool for fungal endophthalmitis. ${ }^{12}$ Although PCR does not replace conventional mycologic methods, the main advantages of PCR are higher sensitivity and rapid results. However, because of the high cost and equipment requirements, PCR has limited clinical application.

Our study demonstrated that histopathological techniques to detect pathogenic fungi from clinical vitreous specimens could lead to a diagnosis in most suspected fungal endophthalmitis cases according to the usual standards that are accepted for patient care even though they are not able to determine the strain in ocular samples. As an area for future development of vitreous diagnostic tests, large numbers of specimens are required for further study.

The combined use of histopathological detection, culture, and smear increased the fungi detection rate significantly and should, therefore, be considered as the microbiological method of choice in the work-up of fungal endophthalmitis.

\section{Summary}

What was known before

- Conventional diagnostic techniques and investigative procedures for the diagnosis of fungal endophthalmitis, such as smear or culture, from intraocular fluids (aqueous humor or vitreous) can suffer from poor sensitivity (25-60\%).

What this study adds

- In this study, we report the histopathological detection results in vitreous biopsy fluid from patients who underwent diagnostic vitrectomy for clinically suspected fungal endophthalmitis for the first time.

\section{Conflict of interest}

The authors declare no conflict of interest. 


\section{References}

1 Chakrabarti A, Shivaprakash MR, Singh R, Tarai B, George VK, Fomda BA et al. Fungal endophthalmitis: fourteen years' experience from a center in india. Retina 2008; 28: 1400-1407.

2 Edwards Jr, JE, Foos RY, Montgomerie JZ, Guze LB. Ocular manifestations of candida septicemia: review of seventy-six cases of hematogenous candida endophthalmitis. Medicine 1974; 53: 47-75.

3 Rao NA, Hidayat AA. Endogenous mycotic endophthalmitis: variations in clinical and histopathologic changes in candidiasis compared with aspergillosis. Am J Ophthalmol 2001; 132: 244-251.

4 Kunimoto DY, Das T, Sharma S, Jalali S, Majji AB, Gopinathan $\mathrm{U}$ et al. Microbiologic spectrum and susceptibility of isolates: Part i. Postoperative endophthalmitis. Endophthalmitis research group. Am J Ophthalmol 1999; 128: 240-242.

5 Sharma S, Jalali S, Adiraju MV, Gopinathan U, Das T. Sensitivity and predictability of vitreous cytology, biopsy, and membrane filter culture in endophthalmitis. Retina 1996; 16: 525-529.

6 Malinowski SM. The vitreous trap: a simple, surgeoncontrolled technique for obtaining undiluted vitreous and subretinal specimens during pars plana vitrectomy. Retina 2010; 30: 828-829.

7 Davis JL, Miller DM, Ruiz P. Diagnostic testing of vitrectomy specimens. Am J Ophthalmol 2005; 140: 822-829.

8 Marticorena J, Romano V, Gomez-Ulla F. Sterile endophthalmitis after intravitreal injections. Mediat Inflamm 2012; 2012: 928123.

9 Arevalo JF. Retinal and Choroidal Manifestations of Selected Systemic Diseases. 1st edn. Springer: New York, NY, USA, 2012.

10 Binder MI, Chua J, Kaiser PK, Procop GW, Isada CM. Endogenous endophthalmitis: an 18-year review of culture-positive cases at a tertiary care center. Medicine 2003; 82: 97-105.

11 Barza M, Pavan PR, Doft BH, Wisniewski SR, Wilson LA, Han DP et al. Evaluation of microbiological diagnostic techniques in postoperative endophthalmitis in the endophthalmitis vitrectomy study. Arch Ophthalmol 1997; 115: 1142-1150.

12 Bharathi MJ, Rameshkumar G, Ramakrishnan R, Venugopal Reddy YC, Shivkumar C, Ramesh S. Comparative evaluation of uniplex, nested, semi-nested, multiplex and nested multiplex PCR methods in the identification of microbial etiology of clinically suspected infectious endophthalmitis. Curr Eye Res 2013; 38: 550-562. 